\title{
Social Prescribing
}

\section{Hans Stalder}

Prof. Dr. med., Mitglied der Redaktion

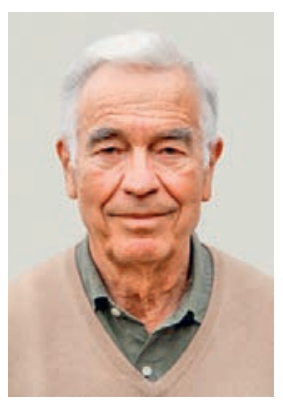

The number of annual deaths attributable to low social support was similar to the number from lung cancer [1].

Die Definition der Gesundheit der WHO - ein «Zustand vollständigen physischen, mentalen und sozialen Wohlbefindens» - hatte den Vorteil, selbst wenn diese Definition heute in Frage gestellt wird - soziales Wohlbefinden auf dieselbe Ebene zu stellen wie physische und psychische Parameter. Für letztere verfügen wir heute über fundierte Diagnose- und Behandlungsmethoden; dies gilt jedoch nicht für die sozialen Determinanten. Wir können die Lipide oder den Blutdruck messen oder eine Depression diagnostizieren. Die Frage, wie wir mit sozialen Risikofaktoren umgehen, sie untersuchen oder gar behandeln, wurde bislang nur wenig untersucht, obwohl seit langem bekannt ist, dass ihnen deutlich mehr Bedeutung zukommt als vielen biologischen Faktoren [2] - eine Tatsache, die auch in der Schweiz bestätigt wurde [3].

Wir wissen beispielsweise, dass niedrige Schulausbildung und Armut mit schlechter Gesundheit assoziiert sind, aber wir haben dafür keinen pathologischen Grenzwert definiert wie beispielsweise für das Cholesterin oder den Blutdruck. Weitere Faktoren wie Lebensraum, Umweltverschmutzung oder Lärm sind noch schwieriger zu identifizieren oder zu quantifizieren. Immerhin wurden verschiedene Fragebögen entwickelt, um ein soziales Profil für einzelne Patienten zu erstellen [4].

Wenn die sozioökonomischen Faktoren so wichtig sind, sollten wir auch diese optimieren können. Natürlich ist dies zuallererst Aufgabe der Gesundheitspolitik. Sie muss Armut und Diskriminierung mindern, den Zugang zum Gesundheitswesen fördern, die Gesundheitsbildung verbessern etc. Aber sollte sich nicht auch jeder Arzt um die sozialen Faktoren jeder seiner Patienten kümmern und sie verbessern? Das ist die Idee des Social Prescribing [5].

Social Prescribing ist definiert als "a mechanism for linking patients with non-medical sources of support within the community" [5]. Der britische Nationale Gesundheitsdienst (NHS) betrachtet das als eine Priorität [6]. Da nicht jeder Allgemeinmediziner in diesem Bereich spezialisiert ist, wurden "Link Workers» eingeführt. Sie sind im Sozialbereich spezialisiert und entsprechen bei uns in etwa den Sozialarbeitern. Die Interventionen können zahlreich und vielfältiger Natur sein. Dazu zählen u.a. die bei uns bekannte therapeutische Schulung, aber auch andere Aktivitäten wie beispielsweise die Unterstützung bei der Begleichung von Rechnungen oder Schuldnerhilfe, die Entfernung von Teppichen, über die man stolpern könnte, das Auffinden von sozialen Aktivitäten für vereinsamte Menschen, die Förderung physischer Aktivitäten, juristischer Beistand, die Organisation eines Sprachkurses ... Ein Grossteil dieser Aktivitäten sind nicht teuer, und manche werden von Freiwilligen ausgeführt. Sie sind individuell auf den Bedarf des einzelnen Patienten abgestimmt: Das ist personalisierte Medizin - nicht um das optimale Arzneimittel ausfindig zu machen, sondern individuelle soziale Defizite aufzuspüren und zu ergänzen.

Die Idee ist attraktiv. Wie auch im Bereich der personalisierten Biomedizin stehen wir am Anfang einer vielversprechenden Entwicklung. Momentan deuten einige Untersuchungen und Metaanalysen zum Thema Social Prescribing auf die Effizienz solcher Interventionen - allerdings noch nicht definitiv [5, 7]. Der NHS steckt viel Geld in die Sache und geht davon aus, dass künftig nahezu eine Million Patienten in Grossbritannien davon profitieren könnten. Ähnliche Entwicklungen gibt es auch in anderen Ländern [6]. Wir sollten die Evolution dieses neuen Therapiesektors aufmerksam verfolgen und - warum nicht? - an seiner Fortentwicklung und Bewertung mitwirken. Schon jetzt sollte auch eine Sozialarbeiterin Teil unserer interprofessionellen Teams sein!

Literatur

1 Daniel H, Bornstein SS, Kane GC; Health and Public Policy Committee of the American College of Physicians. Addressing Social Determinants to Improve Patient Care and Promote Health Equity: An American College of Physicians Position Paper. Ann Intern Med. 2018;168:577-8.

2 Marmot MG, Rose G, Shipley M, Hamilton PJ. Employment grade and coronary heart disease in British civil servants. J Epidemiol Community Health. 1978;32:244-9.

3 https://www.bfs.admin.ch/bfs/de/home/statistiken/ gesundheit/determinanten/soziale-situation.html

4 LaForge K, Gold R, Cottrell E, Bunce AE, Proser M, Hollombe C, Dambrun K, Cohen DJ, Clark KD. How 6 organizations developed tools and processes for social determinants of health screening in primary care: An overview. J Ambul Care Manage. 2018;41:2-14.

5 Chatterjee HJ, CamicPM, Lockyerb B, Thomson LJM. Non-clinical community interventions of social prescribing schemes: a systematised review. Arts \& Health. 2018;10:97-123.

6 Drinkwater C, Wildman J, Moffatt S. Social prescribing. BMJ. 2019;364:1285-9.

7 Gottlieb LM, Wing H, Adler NE. A systematic review of interventions on patients' social and economic needs. Am J Prev Med 2017;53:719-29. 